\title{
WHAT'S IN A NAME? A SYSTEMS THINKING FRAMEWORK FOR RESOURCE TYPES IN NONPROFIT MARKETING
}

\author{
Erica Brady, Monash University, Australia \\ Linda Brennan, RMIT, Vietnam
}

\begin{abstract}
This paper presents a framework that helps researchers and practitioners to consider the types and nature of resources in the not for profit domain. The framework draws from the existing literature and includes theoretical and practical considerations. Measurement of outcomes in the third sector is problematic, often due to the multiplicity of theoretical domains contributing to the sector. Integral to the third sector is the need to maximize the use of scarce resources to achieve social good, activities that can extend well beyond that of fundraising.

Whilst the application of segmentation is still debated within some areas of the sector, recent research supports its use. Interactants in the NPO exchange system will each have differing motivations for participating in the exchange. However, typing by motivation is a form of 'micro' segmentation. While micro-segmentation is important for attracting a particular individual or designing a promotion strategy, this does not assist in broader thinking about what 'customers' want and therefore what are the appropriate resource acquisition strategies. The dilemma for the NPO is how to identify, not only the motivations, but to also calculate the transaction costs for the interactants in the system.
\end{abstract}

For the purposes of clarity, in this paper we are only interested in 'customers' who contribute to the resources of an organization, and therefore are not at the receiving end of the resource system (beneficiaries or recipients). Extant work considers 'customers' in categories such as 'volunteer', 'donor', 'philanthropist', however we argue that these labels can be misleading when trying to segment the resource 'market' as each of these labels have connotations that may not match the broader motivations for contributing to an NPO. Unsurprisingly it is not the demographic characteristics that are the most useful discriminants. For greater applicability and transference across NPO contexts more abstract dimensions are required.

Building on prior literature and discussions with NPO practitioners we established that there are four primary dimensions for considering the resource system of an NPO in terms of inputs and desired outcomes. The first of these is 'Accountability', that is how important it is that the organization accounts for what they do with the resource*. The second is 'Relationship Requirements', which is the level of ongoing contact the giver* wants from the organization. The third is 'Locus of Reward', which refers to the extent to which the giver expects direct benefit or is more altruistic. The final dimension is 'Planning Requirements', which refers to the amount of prior thought, or level of spontaneity, that goes into the resource contribution.

Considering the dimensions of Planning Requirements and Locus of Reward four segments can be identified; Considerates, Deliberates, Responders and Casuals. Consideration of the other two dimensions results in another four segments; Commercials, Shareholders, Believers and Detached.

The development of this typology will help practitioners in two ways. Firstly it provides a targeting tool that allows the NPO to focus their resource acquisition activities on those segments whose broad needs they can readily fulfill. Secondly it provides guidance for the development of strategies to help acquire and satisfy the targeted

References are available by request

*To avoid the connotative problems associated with nomenclature in the area the label 'resource' is being used for what the organization gets and the label 'giver' is being used for the entity that is contributing the resource. 